\title{
BORDERLINE PHYLLODES TUMOUR
}

\section{Saima Haider, Misbah Tasleem}

Department of Radiology, Centre for Nuclear Medicine, Mayo Hospital Lahore, Pakistan

Received: 28 August 2015 / Accepted: 20 September 2015

\begin{abstract}
We report a case of 40-year-old woman with a painful lump in the left breast. Mammography revealed a suspicious mass ACR BI-RADS category 4a. Excision biopsy revealed borderline phyllodes tumour. Our case highlights the significance of multidisciplinary approach in managing benign breast masses such as phyllodes tumours which have malignant potential and a high rate of recurrence.
\end{abstract}

Key words: Breast ultrasound, mammography, phyllodes breast tumour

\section{Introduction}

Phyllodes of the breast is rare fibroepithelial neoplasms and accounts for $<1 \%$ of breast tumours. ${ }^{[1]}$ Accurate pre-operative radiological and pathological diagnosis allows better surgical planning and avoiding unnecessary reoperation. The role of imaging is important in diagnosis and management of phyllodes tumour.

\section{Case Report}

A 40-year-old premenopausal woman presented in a surgical department with acute onset of redness, pain and lump in the left breast. She underwent incision and drainage, and specimen/aspirate was submitted for cytology and culture sensitivity. Gram-positive cocci were isolated and she was treated with antibiotic therapy. Few weeks later, she presented to a recurrent left breast lump rapidly increasing in size. On clinical examination, a fleshy growth with a grape -like appearance was seen with the nipple in the upper and inner quadrant of the left breast [Figure 1]. Mammogram [Figures 2 and 3] showed asymmetry in the left breast with ill-defined radiopaque mass, attached with nipple, protruding on skin.

Ultrasonography [Figures 4 and 5] demonstrated a 2.5-cm multilobulated oval-shaped heterogeneous solid mass in retroareolar region extending medially at 9 O'clock position. Increased vascularity was seen within the mass on colour Doppler ultrasonography

Correspondence: Dr. Saima Haider, Department of Radiology, Centre for Nuclear Medicine, Mayo Hospital Lahore, Pakistan.

Email: saimahaider@gmail.com
[Figures 4]. Excision biopsy was done and histopathology revealed a fibroepithelial tumour of breast i.e., stromal hypercellularity with bundles and fascicles of oval-tospindle cells, mild-to-moderate atypia and 6-7 mitosis/10 HPFs, suggesting borderline phyllodes tumour. [Figure 6] The patient was referred back to the department of surgery for the reassessment for surgery.

\section{Discussion}

Phyllodes tumour was first described in 1838 by Müller as cystosarcoma phyllodes. Later in 1981, the World Health Organisation renamed it as phyllodes and subdivided histologically into benign, borderline and malignant types. ${ }^{[2]}$ Phyllodes tumour has a high recurrence rate of $10-40 \% .^{[3]}$ These tumours are difficult to diagnose clinically; a triple assessment protocol needs to be adopted for timely identification. ${ }^{[4]}$ Careful analysis of radiological features may help in distinguishing from other benign entities such as fibroadenoma, breast abscess and mastitis. Mammography alone is not useful with features as mass, calcifications (macro or micro), combination of both or focal asymmetrical density. Ultrasound images reveal a solid mass with heterogeneous internal echoes without posterior acoustic attenuation. A diagnosis of phyllodes should be considered if sonography reveals fluid-filled elongated spaces or clefts within the mass that represent focal necrosis or degeneration. ${ }^{[5-7]}$ Management of fibroadenomas or benign conditions may be safely done without further investigation. An excisional biopsy or simple enucleation is sufficient. In contrast, phyllodes tumours are progressive lesions that cannot be followed conservatively; wide 


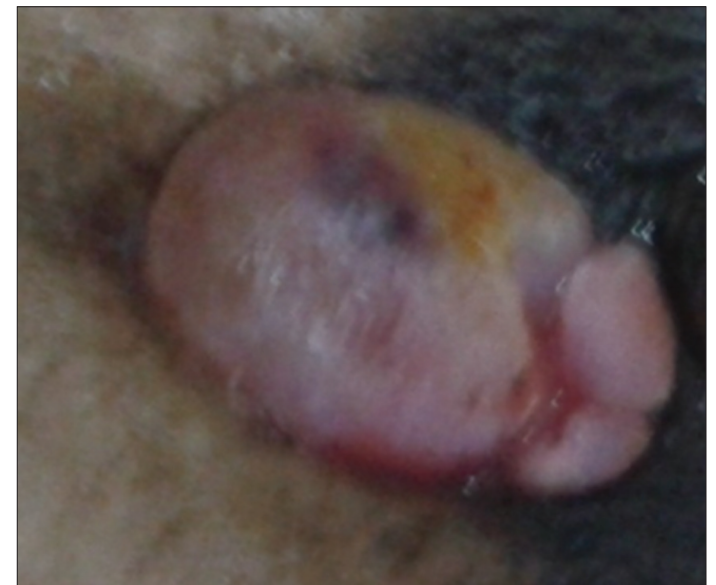

Figure 1: A fleshy growth protruding on skin

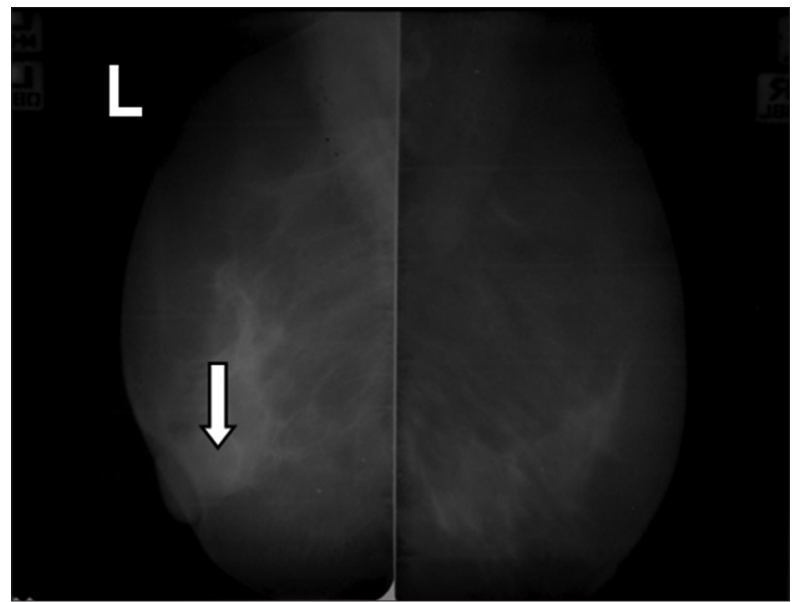

Figure 2: Mediolateral oblique view; asymmetrical radiopacity in left retroaerolar region (arrow)

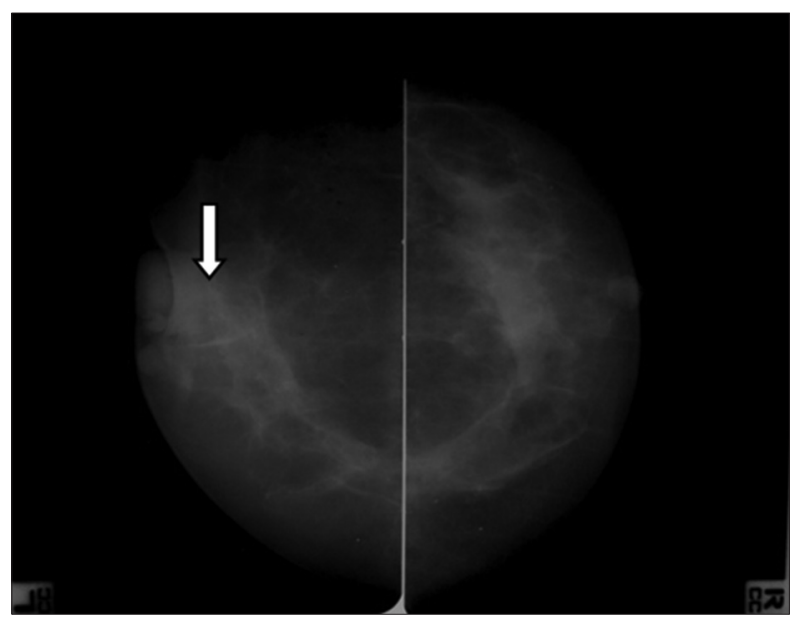

Figure 3: Craniocaudal view; ill-defined radiopaque mass in the left retroaerolar region, protruding on skin (arrow)

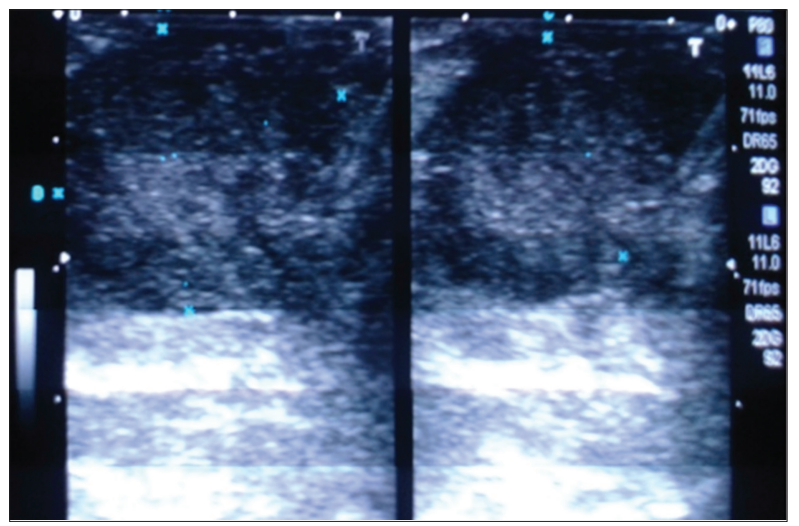

Figure 4: Ultrasound; heterogeneous solid mass $(25 \times 22 \mathrm{~mm})$ with lobulations, attached with skin

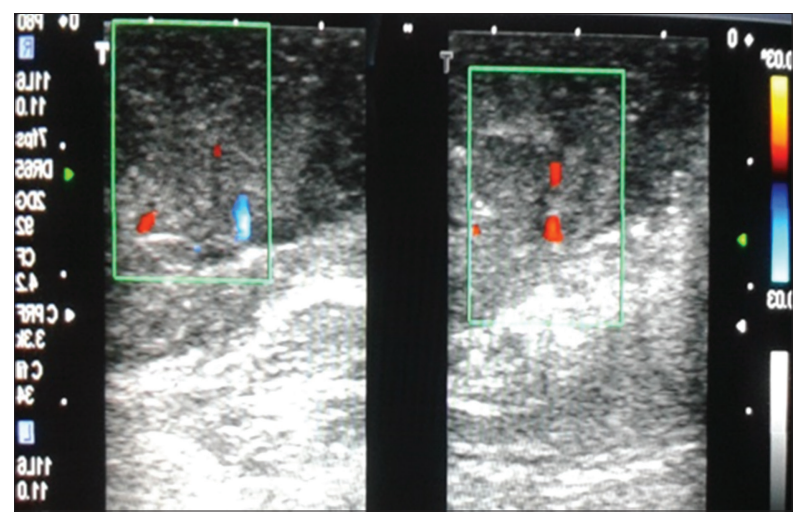

Figure 5: Colour Doppler ultrasound shows vascularity within the mass

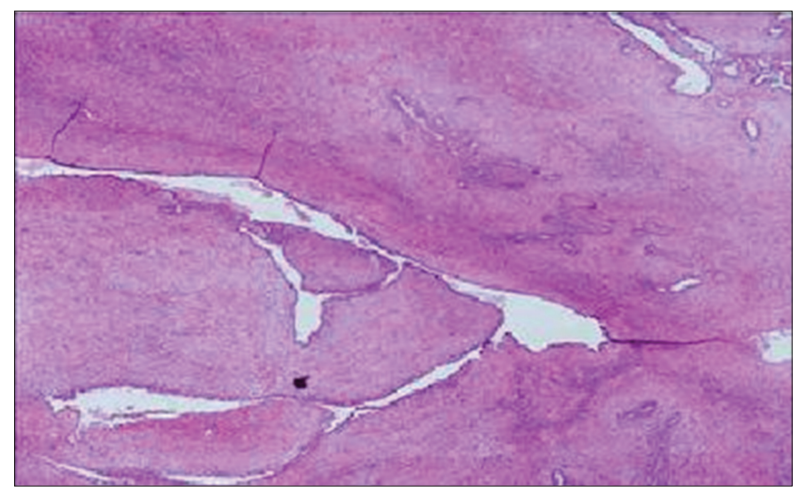

Figure 6: Excision biopsy of left breast lump showed ductal and stromal hypercellularity with bundles and fascicles of oval-tospindle cells with cellular atypia and mitosis

excision with adequate margins is mandatory which can be preventive and curative as well. ${ }^{[8]}$ Early and periodic follow-up with 6 monthly sonographic examinations up to 2 years are recommended after surgery. ${ }^{[9-11]}$ 


\section{Conclusion}

This case highlights that a multidisciplinary approach is essential to distinguish phyllodes from other benign lesions. Clinical findings should be correlated with radiological features for characterisation and pre-surgical planning.

\section{Conflict of Interest}

The authors declare that they have no conflict of interest.

\section{References}

1. Tavassoli FA, Devilee P. Pathology and Genetics of Tumours of the Breast and Femal Genital Organs. France: Lyon: IARC Press; 2003.

2. Histological typing of breast tumors. Second edition. World health organization. Geneva, 1981. Ann Pathol 1982;2:91105.

3. Taira N, Takabatake D, Aogi K, et al. Phyllodes tumour of the breast: Stromal overgrowth and histological classification are useful prognosis-predictive factors for local recurrence in patients with a positive surgical margin.
Jpn J Clin Oncol 2007;37:730-6.

4. Umpleby HC, Guyer PB, Moore I, et al. An evaluation of the preoperative diagnosis and management of cystosarcoma phyllodes. Ann R Coll Surg Engl 1989;71:285-8.

5. Yilmaz E, Sal S, Lebe B. Differetiation of phyllodes tumours versus fibroadenomas: Mammographic and sonographic features. Acta Radiol 2002;43:34-9.

6. Chao TC, Lo YF, Chen SC, et al. Sonographic features of phyllodes tumpours of the breast. Ultrasound Obstet Gynecol 2002;20:64-71.

7. Cole-Beuglet C, Soriano R, Kurtz AB, et al. Ultrasound, $\mathrm{X}$-ray mammography, and histopathology of cystosarcoma phyllodes. Radiology 1983;146:481-6.

8. Parker SJ, Harries SA. Phyllodes tumours: Reviews. Postgrad Med J 2001;77:428-35.

9. Xiao M, Zhu Q, Jiang Y, et al. Local recurrent phyllodes tumours of the breast: Clinical and sonographic features. J Ultrasound Med 2015;34:1631-8.

10. Wiratkapun C, Piyapan P, Lertsithichai P, et al. Fibroadenoma versus phyllodes tumour: Distinguishing factors in patients diagnosed with fibroepithelial lesions after a core needle biopsy. Diagn Interv Radiol 2014;20:27-33.

11. McCarthy E, Kavanagh J, O’Donoghue Y, et al. Phyllodes tumours of the breast: Radiological presentation, management and follow up. Br J Radiol 2014;10:1259. 